\title{
QCD at the FCC-ee
}

\author{
Pier Francesco Monni ${ }^{1, \mathrm{a}}{ }_{\mathbb{D}}$, Giulia Zanderighi $^{2}$ \\ ${ }^{1}$ CERN, Theoretical Physics Department, CH-1211 Geneva 23, Geneva, Switzerland \\ 2 Max-Planck-Institut für Physik, Föhringer Ring 6, 80805 München, Germany
}

Received: 3 August 2021 / Accepted: 23 October 2021

(C) The Author(s) 2021, corrected publication 2022

\begin{abstract}
In this note, we briefly review some theory aspects of Quantum Chromodynamics at the future circular lepton collider (FCC-ee).
\end{abstract}

\section{Introduction}

With its broad programme spanning energies that range from the $Z$ pole to the top-pair threshold, the FCC-ee offers an ideal environment to study several aspects of particle phenomenology. The expected FCC-ee measurements will improve the knowledge of many Higgs and electroweak (EW) parameters with respect to previous colliders by up to one and two orders of magnitude, respectively. Moreover, it will offer great opportunities for the study of the properties of the top quark as well as to explore aspects of flavour physics. In order to use the above measurements to perform consistency tests of the Standard Model (SM) and to derive stringent indirect constraints on new-physics scenarios, it is crucial to reduce theory uncertainties to the level of the experimental ones. This demands unprecedented theoretical accuracy in the strong and the EW sectors of the SM for a variety of processes and observables (see e.g. ref. [1] and references therein), reaching the permille level in some cases. A complementary aspect will be the development of new observables to enhance the sensitivity of the analyses by improving the theoretical control and reducing undesired contamination from background effects. In this brief note, we will discuss some of the advances that will be instrumental in achieving this accuracy on the QCD front, and comment on open challenges towards the desired precision.

\section{Event shapes, jets, and the strong coupling constant}

A crucial theoretical ingredient needed to match the FCC-ee experimental accuracy is the knowledge of SM parameters that enter the calculations. A remarkable phenomenological application on the QCD side is the extraction of the strong coupling constant $\alpha_{s}$ that constitutes the least well-known coupling in the gauge sector of the SM. Currently, the World Average quotes a $\sim 1 \%$ uncertainty in its value $\left(\alpha_{s}\left(M_{Z}\right)=0.1179 \pm 0.0010\right)[2,3]$. The most precise inputs arise from lattice calculations $[3,4]$, whose uncertainties are expected to be reduced by a factor of two in the next decade (see e.g. [5,6] for a recent study).

\footnotetext{
a e-mail: pier.monni@cern.ch (corresponding author)
} 
Conversely, precise determinations of $\alpha_{s}$ at FCC-ee will arguably come from electroweak pseudo observables (EWPO), such as the total cross section $\sigma\left(e^{+} e^{-} \rightarrow\right.$ hadrons) at the $Z$ resonance peak or its ratio to $\sigma\left(e^{+} e^{-} \rightarrow \mu^{+} \mu^{-}\right)$[7]. This quantity is particularly suitable to extract $\alpha_{s}$ due to the small non-perturbative hadronisation corrections that scale with the c.o.m. energy $Q$ as $\left(\Lambda_{\mathrm{QCD}} / Q\right)^{6}$ [8]. With the $\mathcal{O}\left(5 \times 10^{12}\right) Z$ bosons produced at the FCC-ee, the experimental error on $\alpha_{s}$ extractions from fits of the above quantities will be of order $\mathcal{O}(0.00015)$ (see e.g. [7]), hence requiring a substantial reduction in the corresponding theoretical uncertainties. The status of theory computations for such inclusive observables is already very advanced, and QCD corrections are known up to $\mathrm{N}^{4} \mathrm{LO}$ [9-11] ( $\mathrm{N}^{3} \mathrm{LO}$ corrections for massive bottom quarks are also known in a power series in $m_{b}^{2} / Q^{2}$ [12]). Similarly, EW and mixed QCD-EW corrections are available at least up to two loops (see e.g. [13-15] and references therein), and will be discussed elsewhere in this report. Other extractions of $\alpha_{s}$ at the FCC-ee can be derived from $\tau$ decays (see e.g. [3,16,17]) and even $W$ [18] decays, using high-order perturbative QCD computations (see e.g. refs. [9-11,19,20]). However, some outstanding theoretical questions related to the treatment of non-perturbative contributions in fits from $\tau$ decays [21-23], as well as to the difference between extractions relying on contour-improved [24,25] and fixed-order perturbative calculations adopted in the fits are still open. A deeper understanding of these aspects is necessary for robust measurements of $\alpha_{s}$ from FCC-ee data. Furthermore, the study of these decays at the FCC-ee will be instrumental to explore aspects of flavour physics and lepton flavour universality (see e.g. [26,27] and references therein).

Finer details of strong dynamics can be explored through event shapes, designed to study the geometrical properties of hadronic events, or jet rates that, within a specific jet-clustering algorithm, allow one to classify the event in terms of its jet multiplicity. Owing to their sensitivity to QCD radiation, they are widely used to measure $\alpha_{s}$, and to calibrate nonperturbative hadronisation models (see e.g. ref. [28] and references therein). Moreover, their relative simplicity allows for very accurate predictions. Fully differential calculations for the process $e^{+} e^{-} \rightarrow Z / \gamma^{*} \rightarrow q \bar{q}+X$ at $\mathrm{N}^{3} \mathrm{LO}\left(\alpha_{s}^{3}\right)$ in QCD for massless partons in the final state can be derived starting from the results of refs. [29-33] with the inclusive cross section at $\mathrm{N}^{3} \mathrm{LO}$ [19]. Similarly, the production of heavy (notably bottom) quarks $e^{+} e^{-} \rightarrow Z / \gamma^{*} \rightarrow Q \bar{Q}+X$ can be described at NNLO in QCD using the predictions of refs. [34-36] and [12]. For higher jet-multiplicities, the computation of QCD radiative corrections with massless final-state partons has been pushed to NLO for $e^{+} e^{-} \rightarrow Z / \gamma^{*} \rightarrow n$ jets with $n=5$ [37], and $n=6,7$ [38]. The perturbative description of kinematical regimes that require the all-order resummation of radiative corrections has also improved substantially in the past decade, and the state-of-the-art calculations for standard global event shapes and jet rates in $e^{+} e^{-} \rightarrow Z / \gamma^{*} \rightarrow q \bar{q}+X$ are either NNLL or $\mathrm{N}^{3}$ LL [39-55]. Accurate predictions can be also obtained for $e^{+} e^{-} \rightarrow Z / \gamma^{*} \rightarrow q \bar{q} g+X$ observables [56-59], particularly important for the study of gluon-initiated jets (and corresponding tuning of event generators). The computation of non-global observables [60], featuring angular cuts in the final state, poses a major theoretical challenge, and it is currently limited to lower logarithmic accuracy (see e.g. [61-68]). These observables are sensitive to the geometric pattern of soft QCD interference, and are therefore instrumental to our understanding of strong interactions at all orders.

The sensitivity of final-state observables on $\alpha_{s}$ makes them suitable for precise extractions of the strong coupling. Currently, different $\alpha_{s}$ determinations from these observables [3941,69-83] can differ from each other by a few standard deviations. Besides the high-precision perturbative ingredients described above, such fits require input on hadronisation effects. Non-perturbative radiation induces changes in the observable of $\mathcal{O}\left(\Lambda_{\mathrm{QCD}}^{p} / Q^{p}\right)$ (typically 
$p=1$ ) that must be estimated to achieve the desired precision. Aside from the considered observable, the core difference between different $\alpha_{s}$ determinations lies in how one estimates hadronisation corrections. For instance, among the fits currently included in the World Average [3], these corrections are estimated either via Monte Carlo (MC) generators (e.g. in $[71,73,75,79,82]$ ) or with analytic models $[40,72,76,78]$ (based for instance on dispersive methods or shape functions [8,84-95]).

In this context, the use of event generators is sometimes criticised on the ground that they are tuned on less accurate perturbative calculations, and therefore the separation between perturbative and non-perturbative components cannot easily be related to today's highestaccuracy predictions. Conversely, the analytic models fit simultaneously $\alpha_{s}$ and a nonperturbative model, though considering only the leading term in the expansion of the latter in powers of $\Lambda_{\mathrm{QCD}} / Q$. The coefficient of this correction is extracted from data under the assumption that it does not vary across the spectrum of the event-shape observable used in the fit. Besides the natural question about the size of subleading power corrections, the approximation above leads to systematic uncertainties that affect the $\alpha_{s}$ fits at the few-percent level [96].

Thus, improving our understanding and control over hadronisation corrections is essential for precision at future colliders, and the FCC-ee might contribute in different ways. On the one hand, energies higher than those of previous lepton colliders would arguably justify further development of analytic models based on a power expansion in $\Lambda_{\mathrm{QCD}} / Q$. On the other hand, the energy span and experimental accuracy of FCC-ee will be instrumental to gain better control of non-perturbative dynamics in MC generators, which will be beneficial in all measurements foreseen at FCC-ee, such as $e^{+} e^{-} \rightarrow t \bar{t}, e^{+} e^{-} \rightarrow W^{+} W^{-}$, and $e^{+} e^{-} \rightarrow Z H$. An endeavour of this type will crucially require the development of more accurate MC generators, both regarding the predictions for the hard scattering [97-105] and concerning the formulation of novel parton shower algorithms (both for QCD and QED radiation) that overcome the accuracy limitations of the current designs (see e.g. [106118] for recent work). The resulting improved description of final-state radiation would be instrumental for the development of more accurate modelling of the fragmentation of jets initiated either by light or heavy partons. The understanding of the former is essential for the implementation of quark/gluon jet tagging [119-126] to the study of Higgs production at FCC-ee, while the latter is crucial, for instance, to investigate existing discrepancies in heavyquark observables such as the forward-backward asymmetry [127-130]. Parallel important developments will concern the creation of improved models of colour reconnection, which could be precisely calibrated in reactions like $e^{+} e^{-} \rightarrow W^{+} W^{-} \rightarrow q \bar{q} q^{\prime} \bar{q}^{\prime}$ or $e^{+} e^{-} \rightarrow t \bar{t}$ [131]. In the context of jet observables discussed in this section, one can also envision using accurate $\alpha_{s}$ extractions either from EWPO or from future lattice calculations to model and tune non-perturbative aspects of event generators from differential distributions.

A complementary development would be to design observables with reduced sensitivity to hadronisation, for instance by exploiting the modern knowledge of jet dynamics and their substructure, also via machine learning technology (see e.g. [132] for a review). An example is to groom the final-state event so that non-perturbative corrections are reduced in wellidentified regions of the observable spectrum (see e.g. [133-136]), hence opening promising avenues for complementary extractions of $\alpha_{s}$ [137]. An in-depth study of the effectiveness of these techniques at the energies of the FCC-ee, as well as the estimate of the remaining hadronisation corrections (see e.g. refs. $[137,138]$ for recent studies in specific observables), will be highly desirable in the coming years. 


\section{Top physics}

An essential component of the FCC-ee programme is related to the study of top quarks. These are produced in colour-singlet pairs and are nearly free of background, which arises mainly from $W^{+} W^{-}+$jets production and is non-resonant at collision energies $Q \sim 2 m_{t}$. Therefore, the foreseen threshold scan will lead to extremely precise measurements of top-quark properties such as its mass, width and $Z / \gamma t \bar{t}$ couplings, and will allow indirect constraints of the top Yukawa $\left(y_{t}\right)$, which will be already known with $\sim 3 \%$ accuracy after HL-LHC. These quantities are a central element of global fits, and the augmented precision will increase the sensitivity to indirect effects of new physics.

The most precise measurements of the top mass at hadron colliders rely on its direct reconstruction from kinematical distributions of the top decay in $t \bar{t}$ events. Today's stateof-the-art determination quotes a total error of about $500 \mathrm{MeV}$ [3], and the experimental uncertainties will be reduced further after HL-LHC $[139,140]$. These extractions, however, face significant theoretical limitations due to the complexity of the final state, and to the intrinsic ambiguities that affect the pole mass scheme used in these analyses, related to the presence of infrared renormalons in its definition (see e.g. [141,142] for recent discussions). Conversely, at the FCC-ee, the top mass will be extracted through a precise threshold scan at c.o.m. energies close to $2 m_{t}$, where the top-quark pair is non-relativistic and is subject to Coulomb-type interactions. This allows the definition of short-distance top-mass schemes that are not affected by ambiguities related to infrared physics and are particularly suitable to the production at threshold [143-147]. The clean measurements at the FCC-ee will also enable the comparison of the accurate experimental data to considerably more precise theory predictions than what is available for hadron colliders. The QCD calculations for the lineshape of the $t \bar{t}$ system at threshold $\left(\sigma_{t t}\right)$ reach a remarkable degree of precision. Although NNLO fixed-order QCD calculations are available (see e.g. [148]), in this kinematic regime, $\sigma_{t t}$ receives a substantial contribution from Coulomb corrections due to the non-relativistic nature of the $t \bar{t}$ pair. The latter effects can be accurately described in the context of various effective field theories derived from non-relativistic QCD [149-151], which operate within the power counting $\alpha_{s} \sim v$ (with $v$ being the small velocity of the top quarks). Predictions in this framework are already extremely accurate, and include QCD effects up to $\mathrm{N}^{3} \mathrm{LO}$ [152] (see also refs. $[153,154]$ and references therein for a more detailed discussion), approximate NNLL renormalisation-group improved corrections [155], and the inclusion of EW effects within an analogous EFT framework [156]. In general, only the physical final state $W^{+} W^{-} b \bar{b}+X$ is well-defined in perturbation theory, and therefore one must also include the contribution from (non-resonant) channels that do not involve the creation of a top-quark pair near their mass shell. These require embedding the non-relativistic EFT mentioned above into the unstable particle EFT $[157,158]$, where current predictions reach NNLO accuracy for the non-resonant part (see discussion in ref. [156]). Current projections quote an expected accuracy of about $\Delta m_{t} \sim \mathcal{O}(50) \mathrm{MeV}$ [156] (see also refs. [159,160]) for the top mass in the potential-subtracted scheme [146] from the FCC-ee.

These future measurements will exhibit a parametric sensitivity to both $\alpha_{s}$ and $y_{t}$. To maximize the sensitivity to the top mass and width, such parameters should be preferably obtained from other determinations. These are for instance measurements at the $Z$ peak or lattice simulations of $\alpha_{s}$ (cf. Sect. 2), and direct measurements at HL-LHC of $y_{t}$. For the optimal exploitation of FCC-ee measurements, further theoretical developments are desirable, such as the $\mathrm{N}^{3} \mathrm{LO}$ corrections to the non-resonant channels (currently out of reach) as well as a next-to-leading logarithmic control over the initial state QED radiation, for which progress is currently ongoing (see e.g. [161,162]). 
The theoretical description of differential distributions is less accurate than that of the inclusive quantities just discussed, and reaches either NLO or NNLO only for specific observables $[163,164]$. Thus, further progress is needed in these computations, which are central to control precisely the effect of experimental cuts. Some aspects of such calculations pose considerable theoretical challenges, for instance, concerning the differential calculations in the non-relativistic limit, or the assessment of non-factorisable radiative corrections to the decays of the two top quarks [165]. Another important aspect of differential predictions is that of MC event generators (see e.g. [166]), where theoretical improvement is needed in different directions. A first aspect involves the inclusion of higher-order QCD corrections to the hard scattering, for instance using matching technology along the lines of that developed in recent years at hadron colliders. A second development concerns the accurate description of $t \bar{t}$ production at threshold, for which significant challenges arise from the inclusion of Coulomb corrections discussed in this section within the framework of a fully exclusive event generator. Thirdly, another necessary improvement concerns the parton shower algorithms used to simulate QCD and QED radiation, both for what regards their logarithmic accuracy, as well as regarding the accurate description of radiation off the top-quark decays with a correct account of its finite width. Some aspects of this latter problem are addressed in refs. $[167,168]$ for top quarks produced in hadronic collisions; however, a full description of the hierarchy of scales involved in the process (i.e. $v, m_{t}$, the top width $\Gamma_{t}$ ) is not accounted for by existing algorithms. Finally, the study of non-perturbative power corrections to differential observables is also paramount to carry out a top-quark precision physics programme at the FCC-ee. Here, the accurate extraction of the top mass from the inclusive measurements discussed earlier can arguably be used as input to deepen the understanding of linear renormalon corrections to kinematic distributions (see e.g. ref. [169] for a recent study).

\section{The Higgs sector}

The FCC-ee will operate as a Higgs factory producing at least $10^{6}$ Higgs bosons via the $e^{+} e^{-} \rightarrow Z H$ and $W^{+} W^{-}$fusion $\left(e^{+} e^{-} \rightarrow H \nu \bar{v}\right)$ processes, allowing the precise determination of many Higgs couplings as well as of the Higgs boson width [7]. Besides considerable improvements compared to HL-LHC extractions, this data will be used to constrain some aspects of new physics beyond the SM. Conversely, some important couplings that are not directly accessible at FCC-ee will be only constrained indirectly, as it is the case for the top Yukawa or the trilinear coupling. The top Yukawa will be known at the $3-4 \%$ level after HL-LHC (the improvement on $y_{t}$ foreseen at the FCC-ee is marginal [7]), providing key input to several measurements at the FCC-ee, such as the $t \bar{t}$ threshold scan discussed in Sect. 3. On the other hand, model-independent indirect constraints on the trilinear coupling (see e.g. [170-173]) are expected to reach $\mathcal{O}(40 \%)$ precision, which in combination with HL-LHC will achieve a precision of about $30 \%$ [7]. This coupling will be then determined with an astonishing 5\% precision at FCC-hh, also thanks to the precise knowledge of other Higgs branching ratios and EW couplings gained at FCC-ee. Reaching theoretical uncertainties aligned with the projections for these quantities requires dedicated developments in different areas of both QCD and EW calculations. An active programme focused on the computation of the EW and mixed QCD-EW corrections to the production and decay of a Higgs boson in the above processes is ongoing [174-185], and we refer to these references for a detailed discussion. In the following, we focus on the main pure QCD aspects.

The small background and low hadronic activity of the FCC-ee will allow a detailed study of the hadronic decays of the Higgs boson. Partial widths are currently known at the percent 
level. Specifically, in the case of $H \rightarrow b \bar{b}, \mathrm{~N}^{4} \mathrm{LO}$ QCD corrections are known in the limit of massless bottom quarks $[11,186,187]$, and $\mathrm{N}^{4} \mathrm{LO}$ QCD corrections to $H \rightarrow g g$ have been computed in the heavy-top-mass limit [11]. Besides these partial decay widths, the simulation of Higgs decays at the differential level is paramount either to correct for experimental fiducial acceptances or for the study of differential distributions for angular observables and jet observables measured on the Higgs decays, which are sensitive to quark Yukawa couplings [188,189] or new physics states (see e.g. [190-194]). The future development of heavy and light jet tagging and quark/gluon jet discrimination techniques can be exploited to gain sensitivity to Yukawa couplings to light quarks. This sensitivity can arise both via the decay of the Higgs boson to light, e.g. strange, quarks, ${ }^{1}$ or indirectly via light-quark virtual corrections to the $H \rightarrow g g$ decay. The practical realisation of these ideas relies nevertheless on the assumption that sufficiently accurate theoretical predictions will be achieved to support the measurements. Considerable steps are being taken in this direction. The differential $H \rightarrow b \bar{b}$ decay is known up to $\mathrm{N}^{3} \mathrm{LO}$ [195-200] in the limit of massless $b$ quarks and NNLO (and partially beyond) mass corrections are available [201-208]. Similarly, $H \rightarrow g g$ is known to NNLO in the heavy-top limit, though a $\mathrm{N}^{3} \mathrm{LO}$ calculation for this process is currently within reach. Finite quark mass corrections to $H \rightarrow g g$ are relevant at the level of precision foreseen at FCC-ee, and could be estimated at best at NNLO in QCD in the near future with state-of-the-art calculations (see e.g. [202,209-212]). Calculations of hadronic event shapes and jet resolutions at NLO [213-215] and NNLO [216] in QCD have also been performed in recent years. As discussed in Sect. 2, the accurate description of these observables also requires the resummation of radiative corrections, which are obtained either by dedicated analytic calculations, largely obtained with the same techniques used in the context of $e^{+} e^{-} \rightarrow Z \rightarrow$ hadrons, or using exclusive event generators matched to parton showers [217-219]. Interesting theoretical challenges are posed by the consistent description of the radiation off massive quark loops at all perturbative orders (entering for instance $H \rightarrow g g$, as discussed, e.g. in refs. [220-226]) and whose understanding can play a crucial role in new physics scenarios that modify the quark Yukawa couplings (see e.g. refs. [227, 228] for a discussion at hadron colliders). Finally, a special role is again played by the modelling of non-perturbative corrections in hadronic final states that, due to the relatively low energy of the decay $\left(\sim m_{H}\right)$, will have a sizeable impact on most differential measurements of the (hadronic) Higgs decay products. In this respect, the same considerations made in Sect. 2 apply, and the study of the Higgs boson at the FCC-ee will benefit enormously from future developments in the modelling of such effects evoked in Sect. 2.

The FCC-ee will also allow for the exploration of rare and exotic decays of the Higgs boson, such as $H \rightarrow Z \gamma, \mu \mu$ and $\mu \tau$ (the latter being forbidden in the SM). Experimental constraints on $H \rightarrow Z \gamma, \mu \mu$ will be already set at the $10 \%$ level at HL-LHC. While the improvement on these constraints reached at the sole FCC-ee will be marginal, the whole FCC programme will drastically reduce the uncertainty on the measurement of such couplings [229]. In addition, valuable information can be extracted from exclusive decays to mesons, which are sensitive to the Yukawa couplings to second- and first-generation quarks. Examples are the decays into $J / \psi, \phi, \rho$ and $\omega$, in association with either a photon or a weak vector boson [230-234]. Accurate predictions for these decays in the framework of QCD factorisation are

\footnotetext{
1 In optimistic scenarios constraints on first-generation Yukawa couplings can be obtained [188].
} 
available (see e.g. [230,235-237] and references therein). However, the tiny branching ratios will make the measurements very challenging due to the limited statistics at FCC-ee [7].

Funding Open access funding provided by CERN (European Organization for Nuclear Research). This project is co-funded from the European Union's Horizon 2020 research and innovation programme under grant agreement No 95175.

Open Access This article is licensed under a Creative Commons Attribution 4.0 International License, which permits use, sharing, adaptation, distribution and reproduction in any medium or format, as long as you give appropriate credit to the original author(s) and the source, provide a link to the Creative Commons licence, and indicate if changes were made. The images or other third party material in this article are included in the article's Creative Commons licence, unless indicated otherwise in a credit line to the material. If material is not included in the article's Creative Commons licence and your intended use is not permitted by statutory regulation or exceeds the permitted use, you will need to obtain permission directly from the copyright holder. To view a copy of this licence, visit http://creativecommons.org/licenses/by/4.0/.

\section{References}

1. A. Freitas et al., Theoretical uncertainties for electroweak and Higgs-boson precision measurements at FCC-ee, arXiv:1906.05379

2. J. Huston, K. Rabbertz, G. Zanderighi, 2019 update to the quantum Chromodynamics review, 2019

3. PARticle Data Group collaboration, P. Zyla et al., Review of Particle Physics, PTEP 2020 (2020) $083 \mathrm{C} 01$

4. Flavour Lattice Averaging Group collaboration, S. Aoki et al., FLAG Review 2019: Flavour Lattice Averaging Group (FLAG), Eur. Phys. J. C 80 (2020) 113, [arXiv:1902.08191]

5. M. Dalla Brida, A. Ramos, The gradient flow coupling at high-energy and the scale of SU(3) Yang-Mills theory. Eur. Phys. J. C 79, 720 (2019). [arXiv:1905.05147]

6. L. Del Debbio, A. Ramos, Lattice determinations of the strong coupling, arXiv:2101.04762

7. FCC collaboration, A. Abada et al., FCC-ee: The Lepton Collider: Future Circular Collider Conceptual Design Report Volume 2, Eur. Phys. J. ST 228 (2019) 261-623

8. Y.L. Dokshitzer, G. Marchesini, B.R. Webber, Dispersive approach to power behaved contributions in QCD hard processes. Nucl. Phys. B 469, 93-142 (1996). [arXiv:hep-ph/9512336]

9. P.A. Baikov, K.G. Chetyrkin, J.H. Kuhn, Order alpha**4(s) QCD Corrections to Z and tau Decays. Phys. Rev. Lett. 101, 012002 (2008). [arXiv:0801.1821]

10. P.A. Baikov, K.G. Chetyrkin, J.H. Kuhn, J. Rittinger, Complete $\mathcal{O}\left(\alpha_{s}^{4}\right)$ QCD Corrections to Hadronic Z-Decays. Phys. Rev. Lett. 108, 222003 (2012). [arXiv:1201.5804]

11. F. Herzog, B. Ruijl, T. Ueda, J.A.M. Vermaseren, A. Vogt, On Higgs decays to hadrons and the R-ratio at $N^{4}$ LO. JHEP 08, 113 (2017). [arXiv:1707.01044]

12. K.G. Chetyrkin, R.V. Harlander, J.H. Kühn, Quartic mass corrections to $R_{\text {had }}$ at order $\alpha^{3}(s)$. Nucl. Phys. B 586, 56-72 (2000). [arXiv:hep-ph/0005139]

13. I. Dubovyk, A. Freitas, J. Gluza, T. Riemann, J. Usovitsch, Complete electroweak two-loop corrections to Z boson production and decay. Phys. Lett. B 783, 86-94 (2018). [arXiv:1804.10236]

14. I. Dubovyk, A. Freitas, J. Gluza, T. Riemann, J. Usovitsch, Electroweak pseudo-observables and Z-boson form factors at two-loop accuracy. JHEP 08, 113 (2019). [arXiv:1906.08815]

15. L. Chen, A. Freitas, Mixed EW-QCD leading fermionic three-loop corrections at $\mathcal{O}\left(\alpha_{s} \alpha^{2}\right)$ to electroweak precision observables, arXiv:2012.08605

16. A. Pich, A. Rodríguez-Sánchez, Determination of the QCD coupling from ALEPH $\tau$ decay data. Phys. Rev. D 94, 034027 (2016). [arXiv:1605.06830]

17. D. Boito, M. Golterman, K. Maltman, S. Peris, Strong coupling from hadronic $\tau$ decays: A critical appraisal. Phys. Rev. D 95, 034024 (2017). [arXiv:1611.03457]

18. D. d'Enterria, M. Srebre, $\alpha_{s}$ and $\mathrm{V}_{\mathrm{cs}}$ determination, and CKM unitarity test, from $\mathrm{W}$ decays at NNLO. Phys. Lett. B 763, 465-471 (2016). ([arXiv:1603.06501])

19. S.G. Gorishnii, A.L. Kataev, S.A. Larin, The $O\left(\alpha_{s}^{3}\right)$-corrections to $\sigma_{\text {tot }}\left(e^{+} e^{-} \rightarrow\right.$ hadrons $)$ and $\Gamma\left(\tau^{-} \rightarrow v_{\tau}+\right.$ hadrons $)$ in QCD. Phys. Lett. B 259, 144-150 (1991)

20. K.G. Chetyrkin, J.H. Kuhn, Quadratic mass corrections of order O (alpha(s)**3 m(q)**2/s) to the decay rate of Z and W bosons. Phys. Lett. B 406, 102-109 (1997). [arXiv:hep-ph/9609202]

21. A. Pich, Precision Tau Physics. Prog. Part. Nucl. Phys. 75, 41-85 (2014). [arXiv:1310.7922] 
22. M. Davier, A. Höcker, B. Malaescu, C.-Z. Yuan, Z. Zhang, Update of the ALEPH non-strange spectral functions from hadronic $\tau$ decays. Eur. Phys. J. C 74, 2803 (2014). [arXiv:1312.1501]

23. D. Boito, M. Golterman, K. Maltman, J. Osborne, S. Peris, Strong coupling from the revised ALEPH data for hadronic $\tau$ decays. Phys. Rev. D 91, 034003 (2015). [arXiv:1410.3528]

24. A.A. Pivovarov, Renormalization group analysis of the tau lepton decay within QCD. Sov. J. Nucl. Phys. 54, 676-678 (1991). [arXiv:hep-ph/0302003]

25. F. Le Diberder, A. Pich, The perturbative QCD prediction to R(tau) revisited. Phys. Lett. B 286, 147-152 (1992)

26. A. Pich, Precision physics with inclusive QCD processes, Prog. Part. Nucl. Phys. 117 (2021) 103846, [2012.04716]

27. A. Pich, Challenges for tau physics at the TeraZ, 2012.07099

28. M. Dasgupta, G.P. Salam, Event shapes in e+ e- annihilation and deep inelastic scattering. J. Phys. G 30, R143 (2004). ([hep-ph/0312283])

29. A. Gehrmann-De Ridder et al., NNLO corrections to event shapes in $e^{+} e^{-}$annihilation, JHEP 12 (2007) 094 [0711.4711]

30. A. Gehrmann-De Ridder et al., Jet rates in electron-positron annihilation at $O\left(\alpha_{S}^{3}\right)$ in QCD, Phys. Rev. Lett. 100 (2008) 172001, [0802.0813]

31. S. Weinzierl, NNLO corrections to 3-jet observables in electron-positron annihilation. Phys. Rev. Lett. 101, 162001 (2008). ([0807.3241])

32. S. Weinzierl, Event shapes and jet rates in electron-positron annihilation at NNLO. JHEP 06, 041 (2009). ([0904.1077])

33. V. Del Duca et al., Jet production in the CoLoRFulNNLO method: event shapes in electron-positron collisions, Phys. Rev. D94 (2016) 074019, [1606.03453]

34. W. Bernreuther, A. Brandenburg, P. Uwer, Next-to-leading order QCD corrections to three jet crosssections with massive quarks. Phys. Rev. Lett. 79, 189-192 (1997). ([hep-ph/9703305])

35. P. Nason, C. Oleari, Next-to-leading order corrections to momentum correlations in $Z^{0} \rightarrow b \bar{b}$. Phys. Lett. B 407, 57-60 (1997). ([hep-ph/9705295])

36. P. Nason, C. Oleari, Next-to-leading order corrections to the production of heavy flavor jets in $e^{+} e^{-}$ collisions. Nucl. Phys. B 521, 237-273 (1998). ([hep-ph/9709360])

37. R. Frederix, S. Frixione, K. Melnikov, G. Zanderighi, NLO QCD corrections to five-jet production at LEP and the extraction of $\alpha_{S}\left(M_{Z}\right)$. JHEP 11, 050 (2010). ([1008.5313])

38. S. Becker, D. Goetz, C. Reuschle, C. Schwan, S. Weinzierl, NLO results for five, six and seven jets in electron-positron annihilation. Phys. Rev. Lett. 108, 032005 (2012). ([1111.1733])

39. T. Becher, M. Schwartz, A precise determination of $\alpha_{s}$ from LEP thrust data using effective field theory. JHEP 07, 034 (2008). ([0803.0342])

40. R. Abbate et al., Thrust at $N^{3} \mathrm{LL}$ with power corrections and a precision global fit for $\alpha_{S}\left(M_{Z}\right)$. Phys. Rev. D 83, 074021 (2011). ([1006.3080])

41. Y.-T. Chien, M.D. Schwartz, Resummation of heavy jet mass and comparison to LEP data, JHEP 1008 (2010) 058 [1005.1644]

42. P.F. Monni, T. Gehrmann, G. Luisoni, Two-Loop Soft Corrections and Resummation of the Thrust Distribution in the Dijet Region. JHEP 08, 010 (2011). ([1105.4560])

43. T. Becher, G. Bell, NNLL Resummation for jet broadening. JHEP 1211, 126 (2012). ([1210.0580])

44. V. Mateu, G. Rodrigo, Oriented Event Shapes at $N^{3} \mathrm{LL}+O\left(\alpha_{S}^{2}\right)$. JHEP 11, 030 (2013). ([1307.3513])

45. A. Hoang et al., $C$-parameter distribution at $N^{3} \mathrm{LL}^{\prime}$ including power corrections. Phys. Rev. D 91, 094017 (2015). ([1411.6633])

46. D. de Florian, M. Grazzini, The back-to-back region in $e^{+} e^{-}$energy-energy correlation. Nucl. Phys. B 704, 387-403 (2005). ([hep-ph/0407241])

47. A. Banfi et al., A general method for the resummation of event-shape distributions in $e^{+} e^{-}$annihilation. JHEP 1505, 102 (2015). ([1412.2126])

48. A. Banfi et al., The two-jet rate in $e^{+} e^{-}$at next-to-next-to-leading-logarithmic order, Phys. Rev. Lett. 117 (2016) 172001, [1607.03111]

49. Z. Tulipánt, A. Kardos, G. Somogyi, Energy-energy correlation in electron-positron annihilation at NNLL + NNLO accuracy. Eur. Phys. J. C 77, 749 (2017). ([1708.04093])

50. I. Moult, H.X. Zhu, Simplicity from Recoil: The Three-Loop Soft Function and Factorization for the Energy-Energy Correlation. JHEP 08, 160 (2018). ([1801.02627])

51. A. Banfi, B.K. El-Menoufi, P.F. Monni, The Sudakov radiator for jet observables and the soft physical coupling, 1807.11487

52. G. Bell et al., $e^{+} e^{-}$angularity distributions at $N N L L^{\prime}$ accuracy, 1808.07867 
53. M. Procura, W.J. Waalewijn, L. Zeune, Joint resummation of two angularities at next-to-next-to-leading logarithmic order, JHEP 10 (2018) 098, [ 1806.10622]

54. M. A. Ebert, B. Mistlberger, G. Vita, The Energy-Energy Correlation in the back-to-back limit at $N^{3} \mathrm{LO}$ and $N^{3} L L^{\prime}, 2012.07859$

55. A. Bris, V. Mateu, M. Preisser, Massive event-shape distributions at $N^{2}$ LL. JHEP 09, 132 (2020). ([2006.06383])

56. S. Catani et al., New clustering algorithm for multi-jet cross-sections in $e^{+} e^{-}$annihilation. Phys. Lett. B 269, 432-438 (1991)

57. A. J. Larkoski, A. Procita, New Insights on an Old Problem: Resummation of the D-parameter, JHEP 02 (2019) 104, [ 1810.06563]

58. H. Chen, M.-X. Luo, I. Moult, T.-Z. Yang, X. Zhang, H. X. Zhu, Three point energy correlators in the collinear limit: symmetries, dualities and analytic results, JHEP 08 (2020) 028, [1912.11050]

59. L. Arpino, A. Banfi , B. K. El-Menoufi, Near-to-planar three-jet events at NNLL accuracy, JHEP 07 (2020) 171, [ 1912.09341]

60. M. Dasgupta, G. Salam, Resummation of nonglobal QCD observables. Phys. Lett. B 512, 323-330 (2001). ([hep-ph/0104277])

61. M. Dasgupta, G.P. Salam, Accounting for coherence in interjet E(t) flow: A Case study. JHEP 03, 017 (2002). ([hep-ph/0203009])

62. A. Banfi, G. Marchesini, G. Smye, Away from jet energy flow. JHEP 08, 006 (2002). ([hep-ph/0206076])

63. H. Weigert, Nonglobal jet evolution at finite N(c). Nucl. Phys. B 685, 321-350 (2004). ([hepph/0312050])

64. Y. Hatta, T. Ueda, Resummation of non-global logarithms at finite $N_{c}$. Nucl. Phys. B 874, 808-820 (2013). ([1304.6930])

65. T. Becher, M. Neubert, L. Rothen, D.Y. Shao, Effective Field Theory for Jet Processes. Phys. Rev. Lett. 116, 192001 (2016). ([1508.06645])

66. A.J. Larkoski, I. Moult, D. Neill, Non-Global Logarithms. Factorization, and the Soft Substructure of Jets, JHEP 09, 143 (2015). ([1501.04596])

67. T. Becher, M. Neubert, L. Rothen, D.Y. Shao, Factorization and Resummation for Jet Processes. JHEP 11, 019 (2016). ([1605.02737])

68. M. Balsiger, T. Becher, D. Y. Shao, NLL' resummation of jet mass. JHEP 04, 020 (2019). ([1901.09038])

69. R.W.L. Jones et al., Theoretical uncertainties on $\alpha_{S}$ from event shape variables in $e^{+} e^{-}$annihilations. JHEP 12, 007 (2003). ([hep-ph/0312016])

70. G. Dissertori et al., First determination of the strong coupling constant using NNLO predictions for hadronic event shapes in $e^{+} e^{-}$annihilations. JHEP 02, 040 (2008). ([0712.0327])

71. JADE collaboration, S. Bethke, S. Kluth, C. Pahl, J. Schieck, Determination of the Strong Coupling alpha(s) from hadronic Event Shapes with $\mathrm{O}($ alpha**3(s)) and resummed QCD predictions using JADE Data, Eur. Phys. J. C64 (2009) 351-360, [0810.1389]

72. R.A. Davison, B.R. Webber, Non-Perturbative Contribution to the Thrust Distribution in e+ e- Annihilation. Eur. Phys. J. C 59, 13-25 (2009). ([0809.3326])

73. G. Dissertori et al., Determination of the strong coupling constant using matched NNLO+NLLA predictions for hadronic event shapes in $e^{+} e^{-}$annihilations. JHEP 08, 036 (2009). ([0906.3436])

74. T. Gehrmann, M. Jaquier, G. Luisoni, Hadronization effects in event shape moments. Eur. Phys. J. C 67, 57-72 (2010). ([0911.2422])

75. OPAL COLlaboration collaboration, G. Abbiendi et al., Determination of $\alpha_{s}$ using OPAL hadronic event shapes at $\sqrt{s}=91-209$ and resummed NNLO calculations, Eur. Phys. J. C71 (2011) 1733, [1101.1470]

76. T. Gehrmann, G. Luisoni, P.F. Monni, Power corrections in the dispersive model for a determination of the strong coupling constant from the thrust distribution. Eur. Phys. J. C 73, 2265 (2013). ([1210.6945])

77. R. Abbate, M. Fickinger, A.H. Hoang, V. Mateu, I.W. Stewart, Precision Thrust Cumulant Moments at $N^{3}$ LL. Phys. Rev. D 86, 094002 (2012). ([1204.5746])

78. A. Hoang et al., Precise determination of $\alpha_{s}$ from the $C$-parameter distribution. Phys. Rev. D 91, 094018 (2015). ([1501.04111])

79. A. Kardos et al., Precise determination of $\alpha_{S}\left(M_{Z}\right)$ from a global fit of energy-energy correlation to NNLO+NNLL predictions. Eur. Phys. J. C 78, 498 (2018). ([1804.09146])

80. G. Dissertori et al., Precise determination of the strong coupling constant at NNLO in QCD from the three-jet rate in electron-positron annihilation at LEP. Phys. Rev. Lett. 104, 072002 (2010). ([0910.4283])

81. JADE COLlaboration collaboration, J. S. et al., Measurement of the strong coupling $\alpha_{S}$ from the three-jet rate in $e^{+} e^{-}$- annihilation using JADE data, Eur. Phys. J. C73 (2013) 2332, [ 1205.3714]

82. A. Verbytskyi, A. Banfi, A. Kardos, P. F. Monni, S. Kluth, G. Somogyi et al., High precision determination of $\alpha_{S}$ from a global fit of jet rates, JHEP 08 (2019) 129, [1902.08158] 
83. A. Kardos, G. Somogyi, A. Verbytskyi, Determination of $\alpha_{S}$ beyond NNLO using event shape moments, 2009.00281

84. G.P. Korchemsky, G.F. Sterman, Nonperturbative corrections in resummed cross-sections. Nucl. Phys. B 437, 415-432 (1995). ([hep-ph/9411211])

85. Y.L. Dokshitzer, A. Lucenti, G. Marchesini, G. Salam, Universality of $1 / \mathrm{Q}$ corrections to jet-shape observables rescued. Nucl. Phys. B 511, 396-418 (1998). ([hep-ph/9707532])

86. M. Beneke, V.M. Braun, L. Magnea, Phenomenology of power corrections in fragmentation processes in e+ e- annihilation. Nucl. Phys. B 497, 297-333 (1997). ([hep-ph/9701309])

87. Y.L. Dokshitzer, A. Lucenti, G. Marchesini, G. Salam, On the universality of the Milan factor for 1/Q power corrections to jet shapes. JHEP 05, 003 (1998). ([hep-ph/9802381])

88. E. Gardi, G. Grunberg, Power corrections in the single dressed gluon approximation: The Average thrust as a case study. JHEP 11, 016 (1999). ([hep-ph/9908458])

89. G.P. Korchemsky, G.F. Sterman, Power corrections to event shapes and factorization. Nucl. Phys. B 555, 335-351 (1999). ([hep-ph/9902341])

90. M. Dasgupta, L. Magnea, G. Smye, Universality of 1/Q corrections revisited. JHEP 11, 025 (1999). ([hep-ph/9911316])

91. G. Salam, D. Wicke, Hadron masses and power corrections to event shapes. JHEP 05, 061 (2001). ([hep-ph/0102343])

92. E. Gardi, Dressed gluon exponentiation. Nucl. Phys. B 622, 365-392 (2002). ([hep-ph/0108222])

93. E. Gardi, L. Magnea, The C parameter distribution in e+ e- annihilation. JHEP 08, 030 (2003). ([hepph/0306094])

94. V. Mateu, I.W. Stewart, J. Thaler, Power Corrections to Event Shapes with Mass-Dependent Operators. Phys. Rev. D 87, 014025 (2013). ([1209.3781])

95. N. Agarwal, A. Mukhopadhyay, S. Pal, A. Tripathi, Power corrections to event shapes using eikonal dressed gluon exponentiation, 2012.06842

96. G. Luisoni, P. F. Monni , G. P. Salam, $C$-parameter hadronisation in the symmetric 3-jet limit and impact on $\alpha_{S}$ fit, 2012.00622

97. S. Frixione, B. Webber, Matching NLO QCD computations and parton shower simulations. JHEP 06, 029 (2002). ([hep-ph/0204244])

98. P. Nason, A new method for combining NLO QCD with shower Monte Carlo algorithms. JHEP 11, 040 (2004). ([hep-ph/0409146])

99. S. Frixione, P. Nason, C. Oleari, Matching NLO QCD computations with Parton Shower simulations: the POWHEG method. JHEP 11, 070 (2007). ([0709.2092])

100. K. Hamilton, P. Nason, C. Oleari, G. Zanderighi, Merging H/W/Z +0 and 1 jet at NLO with no merging scale: a path to parton shower + NNLO matching. JHEP 05, 082 (2013). ([1212.4504])

101. S. Alioli, C.W. Bauer, C. Berggren, F.J. Tackmann, J.R. Walsh, S. Zuberi, Matching Fully Differential NNLO Calculations and Parton Showers. JHEP 06, 089 (2014). ([1311.0286])

102. S. Höche, Y. Li, S. Prestel, Drell-Yan lepton pair production at NNLO QCD with parton showers. Phys. Rev. D 91, 074015 (2015). ([1405.3607])

103. S. Jadach, W. Płaczek, S. Sapeta, A. Siódmok, M. Skrzypek, Matching NLO QCD with parton shower in Monte Carlo scheme - the KrkNLO method. JHEP 10, 052 (2015). ([1503.06849])

104. P.F. Monni, P. Nason, E. Re, M. Wiesemann, G. Zanderighi, MiNNL $O_{P S}$ : a new method to match NNLO QCD to parton showers. JHEP 05, 143 (2020). ([1908.06987])

105. P.F. Monni, E. Re, M. Wiesemann, MiNNL $O_{\mathrm{PS}}$ : optimizing $2 \rightarrow 1$ hadronic processes. Eur. Phys. J. C 80, 1075 (2020). ([ 2006.04133])

106. Z. Nagy, D.E. Soper, A parton shower based on factorization of the quantum density matrix. JHEP 06, 097 (2014). ([1401.6364])

107. S. Höche, F. Krauss, S. Prestel, Implementing NLO DGLAP evolution in Parton Showers, JHEP 10 (2017) 093, [ 1705.00982]

108. R. Ángeles Martínez, M. De Angelis, J. R. Forshaw, S. Plätzer , M. H. Seymour, Soft gluon evolution and non-global logarithms, JHEP 05 (2018) 044, [ 1802.08531]

109. F. Dulat, S. Höche, S. Prestel, Leading-Color Fully Differential Two-Loop Soft Corrections to QCD Dipole Showers, Phys. Rev. D 98 (2018) 074013, [ 1805.03757]

110. M. Dasgupta, F. A. Dreyer, K. Hamilton, P. F. Monni , G. P. Salam, Logarithmic accuracy of parton showers: a fixed-order study, JHEP 09 (2018) 033, [ 1805.09327]

111. G. Bewick, S. Ferrario Ravasio, P. Richardson, M. H. Seymour, Logarithmic accuracy of angular-ordered parton showers, JHEP 04 (2020) 019, [ 1904.11866]

112. J. R. Forshaw, J. Holguin , S. Plätzer, Parton branching at amplitude level, JHEP 08 (2019) 145, [1905.08686] 
113. M. Dasgupta, F. A. Dreyer, K. Hamilton, P. F. Monni, G. P. Salam , G. Soyez, Parton showers beyond leading logarithmic accuracy, Phys. Rev. Lett. 125 (2020) 052002, [2002.11114]

114. J. R. Forshaw, J. Holguin , S. Plätzer, Building a consistent parton shower, JHEP 09 (2020) 014, [2003.06400]

115. K. Hamilton, R. Medves, G. P. Salam, L. Scyboz, G. Soyez, Colour and logarithmic accuracy in final-state parton showers, 2011.10054

116. S. Plätzer, I. Ruffa, Towards Colour Flow Evolution at Two Loops, 2012.15215

117. Z. Nagy, D. E. Soper, Summations by parton showers of large logarithms in electron-positron annihilation, 2011.04777

118. Z. Nagy , D. E. Soper, Summations of large logarithms by parton showers, 2011.04773

119. J. Gallicchio, M.D. Schwartz, Quark and Gluon Tagging at the LHC. Phys. Rev. Lett. 107, 172001 (2011). ([1106.3076])

120. A.J. Larkoski, G.P. Salam, J. Thaler, Energy Correlation Functions for Jet Substructure. JHEP 06, 108 (2013). ([1305.0007])

121. A.J. Larkoski, J. Thaler, W.J. Waalewijn, Gaining (Mutual) Information about Quark/Gluon Discrimination. JHEP 11, 129 (2014). ([1408.3122])

122. B. Bhattacherjee, S. Mukhopadhyay, M.M. Nojiri, Y. Sakaki, B.R. Webber, Associated jet and subjet rates in light-quark and gluon jet discrimination. JHEP 04, 131 (2015). ([1501.04794])

123. P.T. Komiske, E.M. Metodiev, M.D. Schwartz, Deep learning in color: towards automated quark/gluon jet discrimination. JHEP 01, 110 (2017). ([1612.01551])

124. C. Frye, A.J. Larkoski, J. Thaler, K. Zhou, Casimir Meets Poisson: Improved Quark/Gluon Discrimination with Counting Observables. JHEP 09, 083 (2017). ([1704.06266])

125. E.M. Metodiev, J. Thaler, Jet Topics: Disentangling Quarks and Gluons at Colliders. Phys. Rev. Lett. 120, 241602 (2018). ([1802.00008])

126. A.J. Larkoski, E.M. Metodiev, A Theory of Quark vs. Gluon Discrimination, JHEP 10, 014 (2019). ([1906.01639])

127. S. Gori, J. Gu, L.-T. Wang, The $Z b \bar{b}$ couplings at future $e^{+} e^{-}$colliders. JHEP 04, 062 (2016). ([1508.07010])

128. W. Bernreuther, L. Chen, O. Dekkers, T. Gehrmann, D. Heisler, The forward-backward asymmetry for massive bottom quarks at the $Z$ peak at next-to-next-to-leading order QCD. JHEP 01, 053 (2017). ([1611.07942])

129. D. d'Enterria, C. Yan, Revised QCD effects on the $\mathrm{Z} \rightarrow b \bar{b}$ forward-backward asymmetry, 2011.00530

130. B. Yan , C. P. Yuan, The anomalous $Z b \bar{b}$ couplings: From LEP to LHC, 2101.06261

131. J.R. Christiansen, T. Sjöstrand, Color reconnection at future $e^{+} e^{-}$colliders. Eur. Phys. J. C 75, 441 (2015). ([1506.09085])

132. A.J. Larkoski, I. Moult, B. Nachman, Jet Substructure at the Large Hadron Collider: A Review of Recent Advances in Theory and Machine Learning. Phys. Rept. 841, 1-63 (2020). ([1709.04464])

133. C. Frye, A.J. Larkoski, M.D. Schwartz, K. Yan, Factorization for groomed jet substructure beyond the next-to-leading logarithm. JHEP 07, 064 (2016). ([1603.09338])

134. J. Baron, S. Marzani, V. Theeuwes, Soft-Drop Thrust. JHEP 08, 105 (2018). ([1803.04719])

135. A. Kardos, G. Somogyi, Z. Trócsányi, Soft-drop event shapes in electron-positron annihilation at nextto-next-to-leading order accuracy. Phys. Lett. B 786, 313-318 (2018). ([1807.11472])

136. A. Kardos, A.J. Larkoski, Z. Trócsányi, Groomed jet mass at high precision. Phys. Lett. B 809, 135704 (2020). ([2002.00942])

137. S. Marzani, D. Reichelt, S. Schumann, G. Soyez, V. Theeuwes, Fitting the Strong Coupling Constant with Soft-Drop Thrust. JHEP 11, 179 (2019). ([1906.10504])

138. A.H. Hoang, S. Mantry, A. Pathak, I.W. Stewart, Nonperturbative Corrections to Soft Drop Jet Mass. JHEP 12, 002 (2019). ([1906.11843])

139. ATLAS collaboration, Prospects for measurement of the top quark mass using $t \bar{t}$ events with $J / \psi \rightarrow$ $\mu^{+} \mu^{-}$decays with the upgraded ATLAS detector at the High Luminosity LHC

140. CMS collaboration, ECFA 2016: Prospects for selected standard model measurements with the CMS experiment at the High-Luminosity LHC

141. P. Nason, The Top Mass in Hadronic Collisions, pp. 123-151. 2019. 1712.02796. 10.1142/9789813238053_0008

142. A.H. Hoang, What is the Top Quark Mass? Ann. Rev. Nucl. Part. Sci. 70, 225-255 (2020). ([2004.12915])

143. I.I.Y. Bigi, M.A. Shifman, N. Uraltsev, Aspects of heavy quark theory. Ann. Rev. Nucl. Part. Sci. 47, 591-661 (1997). ([hep-ph/9703290])

144. A.H. Hoang, Z. Ligeti, A.V. Manohar, B decays in the upsilon expansion. Phys. Rev. D 59, 074017 (1999). ([hep-ph/9811239]) 
145. A.H. Hoang, Z. Ligeti, A.V. Manohar, B decay and the Upsilon mass. Phys. Rev. Lett. 82, 277-280 (1999). ([hep-ph/9809423])

146. M. Beneke, A Quark mass definition adequate for threshold problems. Phys. Lett. B 434, 115-125 (1998). ([hep-ph/9804241])

147. A. Pineda, Determination of the bottom quark mass from the Upsilon(1S) system. JHEP 06, 022 (2001). ([hep-ph/0105008])

148. L. Chen, O. Dekkers, D. Heisler, W. Bernreuther, Z.-G. Si, Top-quark pair production at next-to-nextto-leading order QCD in electron positron collisions. JHEP 12, 098 (2016). ([1610.07897])

149. B.A. Thacker, G.P. Lepage, Heavy quark bound states in lattice QCD. Phys. Rev. D 43, 196-208 (1991)

150. G.P. Lepage, L. Magnea, C. Nakhleh, U. Magnea, K. Hornbostel, Improved nonrelativistic QCD for heavy quark physics. Phys. Rev. D 46, 4052-4067 (1992). ([hep-lat/9205007])

151. G.T. Bodwin, E. Braaten, G.P. Lepage, Rigorous QCD analysis of inclusive annihilation and production of heavy quarkonium. Phys. Rev. D 51, 1125-1171 (1995). ([hep-ph/9407339])

152. M. Beneke, Y. Kiyo, P. Marquard, A. Penin, J. Piclum, M. Steinhauser, Next-to-Next-to-Next-to-Leading Order QCD Prediction for the Top Antitop $S$-Wave Pair Production Cross Section Near Threshold in $e^{+} e^{-}$Annihilation. Phys. Rev. Lett. 115, 192001 (2015). ([1506.06864])

153. A.H. Hoang et al., Top - anti-top pair production close to threshold: Synopsis of recent NNLO results. Eur. Phys. J. direct 2, 3 (2000). ([hep-ph/0001286])

154. M. Beneke, Y. Kiyo, K. Schuller, Third-order correction to top-quark pair production near threshold I. Effective theory set-up and matching coefficients, 1312.4791

155. A.H. Hoang, M. Stahlhofen, The Top-Antitop Threshold at the ILC: NNLL QCD Uncertainties. JHEP 05, 121 (2014). ([1309.6323])

156. M. Beneke, A. Maier, T. Rauh, P. Ruiz-Femenia, Non-resonant and electroweak NNLO correction to the $e^{+} e^{-}$top anti-top threshold. JHEP 02, 125 (2018). ([1711.10429])

157. M. Beneke, A.P. Chapovsky, A. Signer, G. Zanderighi, Effective theory approach to unstable particle production. Phys. Rev. Lett. 93, 011602 (2004). ([hep-ph/0312331])

158. M. Beneke, A.P. Chapovsky, A. Signer, G. Zanderighi, Effective theory calculation of resonant highenergy scattering. Nucl. Phys. B 686, 205-247 (2004). ([hep-ph/0401002])

159. M. Vos et al., Top physics at high-energy lepton colliders, 1604.08122

160. CLICDP collaboration, H. Abramowicz et al., Top-Quark Physics at the CLIC Electron-Positron Linear Collider, JHEP 11 (2019) 003, [1807.02441]

161. V. Bertone, M. Cacciari, S. Frixione, G. Stagnitto, The partonic structure of the electron at the next-toleading logarithmic accuracy in QED. JHEP 03, 135 (2020). ([1911.12040])

162. S. Frixione, Initial conditions for electron and photon structure and fragmentation functions. JHEP 11, 158 (2019). ([1909.03886])

163. A.H. Hoang, T. Teubner, Top quark pair production close to threshold: Top mass, width and momentum distribution. Phys. Rev. D 60, 114027 (1999). ([hep-ph/9904468])

164. F. Bach, B.C. Nejad, A. Hoang, W. Kilian, J. Reuter, M. Stahlhofen et al., Fully-differential Top-Pair Production at a Lepton Collider: From Threshold to Continuum. JHEP 03, 184 (2018). ([1712.02220])

165. K. Melnikov, O.I. Yakovlev, Final state interaction in the production of heavy unstable particles. Nucl. Phys. B 471, 90-120 (1996). ([hep-ph/9501358])

166. W. Kilian, T. Ohl, J. Reuter, WHIZARD: Simulating Multi-Particle Processes at LHC and ILC. Eur. Phys. J. C 71, 1742 (2011). ([0708.4233])

167. T. Ježo, P. Nason, On the Treatment of Resonances in Next-to-Leading Order Calculations Matched to a Parton Shower. JHEP 12, 065 (2015). ([1509.09071])

168. R. Frederix, S. Frixione, A.S. Papanastasiou, S. Prestel, P. Torrielli, Off-shell single-top production at NLO matched to parton showers. JHEP 06, 027 (2016). ([1603.01178])

169. S. Ferrario Ravasio, P. Nason, C. Oleari, All-orders behaviour and renormalons in top-mass observables. JHEP 01, 203 (2019). ([ 1810.10931])

170. M. McCullough, An Indirect Model-Dependent Probe of the Higgs Self-Coupling. Phys. Rev. D 90, 015001 (2014). ([1312.3322])

171. S. Di Vita, G. Durieux, C. Grojean, J. Gu, Z. Liu, G. Panico et al., A global view on the Higgs self-coupling at lepton colliders. JHEP 02, 178 (2018). ([1711.03978])

172. A. Blondel , P. Janot, Future strategies for the discovery and the precise measurement of the Higgs self coupling, 1809.10041

173. F. Maltoni, D. Pagani, X. Zhao, Constraining the Higgs self-couplings at $e^{+} e^{-}$colliders. JHEP 07, 087 (2018). ([1802.07616])

174. J. Fleischer, F. Jegerlehner, Radiative Corrections to Higgs Production by $e^{+} e^{-} \rightarrow Z H$ in the WeinbergSalam Model. Nucl. Phys. B 216, 469-492 (1983) 
175. B.A. Kniehl, Radiative corrections for associated $Z H$ production at future $e^{+} e^{-}$colliders. Z. Phys. C 55, 605-618 (1992)

176. A. Denner, J. Kublbeck, R. Mertig, M. Bohm, Electroweak radiative corrections to $e^{+} e^{-}--H Z$. Z. Phys. C 56, 261-272 (1992)

177. G. Belanger, F. Boudjema, J. Fujimoto, T. Ishikawa, T. Kaneko, K. Kato et al., Full one loop electroweak radiative corrections to single Higgs production in e+ e-. Phys. Lett. B 559, 252-262 (2003). ([hepph/0212261])

178. A. Denner, S. Dittmaier, M. Roth, M.M. Weber, Electroweak radiative corrections to single Higgs boson production in e+ e- annihilation. Phys. Lett. B 560, 196-203 (2003). ([hep-ph/0301189])

179. F. Boudjema, J. Fujimoto, T. Ishikawa, T. Kaneko, K. Kato, Y. Kurihara et al., Electroweak corrections to Higgs production through ZZ fusion at the linear collider. Phys. Lett. B 600, 65-76 (2004). ([hepph/0407065])

180. Y. Gong, Z. Li, X. Xu, L.L. Yang, X. Zhao, Mixed QCD-EW corrections for Higgs boson production at $e^{+} e^{-}$colliders. Phys. Rev. D 95, 093003 (2017). ([1609.03955])

181. Q.-F. Sun, F. Feng, Y. Jia, W.-L. Sang, Mixed electroweak-QCD corrections to $e^{+} e^{-} \rightarrow H Z$ at Higgs factories. Phys. Rev. D 96, 051301 (2017). ([1609.03995])

182. S. Bondarenko, Y. Dydyshka, L. Kalinovskaya, L. Rumyantsev, R. Sadykov, V. Yermolchyk, Oneloop electroweak radiative corrections to polarized $e^{+} e^{-} \rightarrow$ ZH. Phys. Rev. D 100, 073002 (2019). ([1812.10965])

183. W. Chen, F. Feng, Y. Jia, W.-L. Sang, Mixed electroweak-QCD corrections to $e^{+} e^{-} \rightarrow \mu^{+} \mu^{-} H$ at CEPC with finite-width effect. Chin. Phys. C 43, 013108 (2019). ([1811.05453])

184. Y. Wang, X. Xu, L.L. Yang, Two-loop triangle integrals with 4 scales for the $H Z V$ vertex. Phys. Rev. D 100, 071502 (2019). ([1905.11463])

185. Q. Song, A. Freitas, On the evaluation of two-loop electroweak box diagrams for $e^{+} e^{-} \rightarrow H Z$ production, 2101.00308

186. P.A. Baikov, K.G. Chetyrkin, J.H. Kuhn, Scalar correlator at O(alpha(s)**4), Higgs decay into b-quarks and bounds on the light quark masses. Phys. Rev. Lett. 96, 012003 (2006). ([hep-ph/0511063])

187. J. Davies, M. Steinhauser, D. Wellmann, Completing the hadronic Higgs boson decay at order $\alpha_{s}^{4}$. Nucl. Phys. B 920, 20-31 (2017). ([1703.02988])

188. J. Gao, Probing light-quark Yukawa couplings via hadronic event shapes at lepton colliders. JHEP 01, 038 (2018). ([1608.01746])

189. Q. Bi, K. Chai, J. Gao, Y. Liu , H. Zhang, Investigating Bottom-Quark Yukawa Interaction at Higgs Factory, 2009.02000

190. D.E. Kaplan, M. McEvoy, Searching for Higgs decays to four bottom quarks at LHCb. Phys. Lett. B 701, 70-74 (2011). ([0909.1521])

191. D. Curtin et al., Exotic decays of the $125 \mathrm{GeV}$ Higgs boson. Phys. Rev. D 90, 075004 (2014). ([1312.4992])

192. Z. Liu, L.-T. Wang, H. Zhang, Exotic decays of the $125 \mathrm{GeV}$ Higgs boson at future $e^{+} e^{-}$lepton colliders. Chin. Phys. C 41, 063102 (2017). ([1612.09284])

193. S. Liu, Y.-L. Tang, C. Zhang, S.-H. Zhu, Exotic Higgs Decay $h \rightarrow \phi \phi \rightarrow 4 b$ at the LHeC. Eur. Phys. J. C 77, 457 (2017). ([1608.08458])

194. J. Gao, Higgs boson decay into four bottom quarks in the SM and beyond. JHEP 08, 174 (2019). ([1905.04865])

195. C. Anastasiou, F. Herzog, A. Lazopoulos, The fully differential decay rate of a Higgs boson to bottomquarks at NNLO in QCD. JHEP 03, 035 (2012). ([1110.2368])

196. V. Del Duca, C. Duhr, G. Somogyi, F. Tramontano, Z. Trócsányi, Higgs boson decay into b-quarks at NNLO accuracy. JHEP 04, 036 (2015). ([1501.07226])

197. F. Caola, G. Luisoni, K. Melnikov, R. Röntsch, NNLO QCD corrections to associated $W H$ production and $H \rightarrow b \bar{b}$ decay. Phys. Rev. D 97, 074022 (2018). ([1712.06954])

198. G. Ferrera, G. Somogyi, F. Tramontano, Associated production of a Higgs boson decaying into bottom quarks at the LHC in full NNLO QCD. Phys. Lett. B 780, 346-351 (2018). ([1705.10304])

199. R. Mondini, M. Schiavi, C. Williams, $N^{3}$ LO predictions for the decay of the Higgs boson to bottom quarks. JHEP 06, 079 (2019). ([1904.08960])

200. R. Gauld, A. Gehrmann-De Ridder, E.W.N. Glover, A. Huss, I. Majer, Associated production of a Higgs boson decaying into bottom quarks and a weak vector boson decaying leptonically at NNLO in QCD. JHEP 10, 002 (2019)

201. K.G. Chetyrkin, A. Kwiatkowski, Second order QCD corrections to scalar and pseudoscalar Higgs decays into massive bottom quarks. Nucl. Phys. B 461, 3-18 (1996). ([hep-ph/9505358])

202. S.A. Larin, T. van Ritbergen, J.A.M. Vermaseren, The Large top quark mass expansion for Higgs boson decays into bottom quarks and into gluons. Phys. Lett. B 362, 134-140 (1995). ([hep-ph/9506465]) 
203. R. Harlander, M. Steinhauser, Higgs decay to top quarks at O (alpha-s**2). Phys. Rev. D 56, 3980-3990 (1997). ([hep-ph/9704436])

204. W. Bernreuther, L. Chen, Z.-G. Si, Differential decay rates of CP-even and CP-odd Higgs bosons to top and bottom quarks at NNLO QCD. JHEP 07, 159 (2018). ([1805.06658])

205. A. Primo, G. Sasso, G. Somogyi, F. Tramontano, Exact Top Yukawa corrections to Higgs boson decay into bottom quarks. Phys. Rev. D 99, 054013 (2019). ([1812.07811])

206. A. Behring, W. Bizoń, Higgs decay into massive b-quarks at NNLO QCD in the nested soft-collinear subtraction scheme. JHEP 01, 189 (2020). ([1911.11524])

207. R. Mondini, U. Schubert, C. Williams, Top-induced contributions to $H \rightarrow b \bar{b}$ and $H \rightarrow c \bar{c}$ at $\mathcal{O}\left(\alpha_{s}^{3}\right)$. JHEP 12, 058 (2020). ([2006.03563])

208. A. Behring, W. Bizoń, F. Caola, K. Melnikov, R. Röntsch, Bottom quark mass effects in associated $W H$ production with the $H \rightarrow b \bar{b}$ decay through NNLO QCD. Phys. Rev. D 101, 114012 (2020). ([2003.08321])

209. A. Djouadi, M. Spira, P.M. Zerwas, QCD corrections to hadronic Higgs decays. Z. Phys. C 70, 427-434 (1996). ([hep-ph/9511344])

210. M. Spira, A. Djouadi, D. Graudenz, P.M. Zerwas, Higgs boson production at the LHC. Nucl. Phys. B 453, 17-82 (1995). ([hep-ph/9504378])

211. M. Schreck, M. Steinhauser, Higgs Decay to Gluons at NNLO. Phys. Lett. B 655, 148-155 (2007). ([0708.0916])

212. H. Frellesvig, M. Hidding, L. Maestri, F. Moriello, G. Salvatori, The complete set of two-loop master integrals for Higgs + jet production in QCD. JHEP 06, 093 (2020). ([1911.06308])

213. J. Gao, Y. Gong, W.-L. Ju, L.L. Yang, Thrust distribution in Higgs decays at the next-to-leading order and beyond. JHEP 03, 030 (2019). ([1901.02253])

214. M.-X. Luo, V. Shtabovenko, T.-Z. Yang, H.X. Zhu, Analytic Next-To-Leading Order Calculation of Energy-Energy Correlation in Gluon-Initiated Higgs Decays. JHEP 06, 037 (2019). ([1903.07277])

215. J. Gao, V. Shtabovenko , T.-Z. Yang, Energy-energy correlation in hadronic Higgs decays: analytic results and phenomenology at NLO, 2012.14188

216. R. Mondini, C. Williams, $H \rightarrow b \bar{b} j$ at next-to-next-to-leading order accuracy. JHEP 06, 120 (2019). ([1904.08961])

217. W. Bizoń, E. Re, G. Zanderighi, NNLOPS description of the $H \rightarrow b \bar{b}$ decay with MiNLO. JHEP 06, 006 (2020). ([1912.09982])

218. S. Alioli, A. Broggio, A. Gavardi, S. Kallweit, M. A. Lim, R. Nagar et al., Resummed predictions for hadronic Higgs boson decays, 2009.13533

219. Y. Hu, C. Sun, X.-M. Shen, J. Gao, Hadronic decays of Higgs boson at NNLO matched with parton shower, 2101.08916

220. A. Banfi, P.F. Monni, G. Zanderighi, Quark masses in Higgs production with a jet veto. JHEP 01, 097 (2014). ([1308.4634])

221. M. Grazzini, H. Sargsyan, Heavy-quark mass effects in Higgs boson production at the LHC. JHEP 09, 129 (2013). ([1306.4581])

222. K. Melnikov, A. Penin, On the light quark mass effects in Higgs boson production in gluon fusion. JHEP 05, 172 (2016). ([1602.09020])

223. T. Liu, A.A. Penin, High-Energy Limit of QCD beyond the Sudakov Approximation. Phys. Rev. Lett. 119, 262001 (2017). (1709.01092)

224. T. Liu, A. Penin, High-Energy Limit of Mass-Suppressed Amplitudes in Gauge Theories. JHEP 11, 158 (2018). ([1809.04950])

225. Z. L. Liu, B. Mecaj, M. Neubert, X. Wang, Factorization at Subleading Power and Endpoint Divergences in Soft-Collinear Effective Theory, 2009.04456

226. C. Anastasiou, A. Penin, Light Quark Mediated Higgs Boson Threshold Production in the Next-toLeading Logarithmic Approximation. JHEP 07, 195 (2020). ([2004.03602])

227. F. Bishara, U. Haisch, P.F. Monni, E. Re, Constraining Light-Quark Yukawa Couplings from Higgs Distributions. Phys. Rev. Lett. 118, 121801 (2017). ([1606.09253])

228. Y. Soreq, H.X. Zhu, J. Zupan, Light quark Yukawa couplings from Higgs kinematics. JHEP 12, 045 (2016). ([1606.09621])

229. J. de Blas et al., Higgs Boson studies at future particle colliders. JHEP 1, 139 (2020). ([1905.03764])

230. G.T. Bodwin, F. Petriello, S. Stoynev, M. Velasco, Higgs boson decays to quarkonia and the $H \bar{c} c$ coupling. Phys. Rev. D 88, 053003 (2013). ([1306.5770])

231. A.L. Kagan, G. Perez, F. Petriello, Y. Soreq, S. Stoynev, J. Zupan, Exclusive Window onto Higgs Yukawa Couplings. Phys. Rev. Lett. 114, 101802 (2015). ([1406.1722])

232. D.-N. Gao, A note on Higgs decays into Z boson and $J / \Psi(\Upsilon)$. Phys. Lett. B 737, 366-368 (2014). ([1406.7102]) 
233. T. Modak, R. Srivastava, Probing anomalous Higgs couplings in $\mathrm{H} \rightarrow$ ZV decays. Mod. Phys. Lett. A 32, 1750004 (2017). ([1411.2210])

234. M. König, M. Neubert, Exclusive Radiative Higgs Decays as Probes of Light-Quark Yukawa Couplings. JHEP 08, 012 (2015). ([1505.03870])

235. G.T. Bodwin, H.S. Chung, J.-H. Ee, J. Lee, New approach to the resummation of logarithms in Higgsboson decays to a vector quarkonium plus a photon. Phys. Rev. D 95, 054018 (2017). ([1603.06793])

236. S. Alte, M. König, M. Neubert, Exclusive Weak Radiative Higgs Decays in the Standard Model and Beyond. JHEP 12, 037 (2016). ([1609.06310])

237. N. Brambilla, H.S. Chung, W.K. Lai, V. Shtabovenko, A. Vairo, Order $v^{4}$ corrections to Higgs boson decay into $J / \psi+\gamma$. Phys. Rev. D 100, 054038 (2019). ([1907.06473]) 